\title{
Review of: "A simple method for unsupervised anomaly detection: An application to Web time series data"
}

pengfei jia

Potential competing interests: The author(s) declared that no potential competing interests exist.

This paper proposed a simple anomaly detection method which is used to deal with the unlabeled time series data by using the density ratio estimation based on the state space model. The result implies that it is essential in time series anomaly detection to incorporate the specific information on time series data into the model. When the proposed method is employed to deal with the unlabeled Web time series data, the authors find that the increase in page view caused by e-mail newsletter deliveries is less likely to contribute to completing an insurance contract, and the result also suggests the importance of the simultaneous monitoring of more than one time series. The research of this paper is very interesting. 